\title{
The foramen lacerum: surgical anatomy and relevance for endoscopic endonasal approaches
}

\author{
Wei-Hsin Wang, MD, ${ }^{1,3}$ Stefan Lieber, MD, ${ }^{1}$ Roger Neves Mathias, MD, ${ }^{1}$ Xicai Sun, MD, PhD, ${ }^{1}$ \\ Paul A. Gardner, MD, ${ }^{1}$ Carl H. Snyderman, MD, MBA, ${ }^{2}$ Eric W. Wang, MD, ${ }^{2}$ and \\ Juan C. Fernandez-Miranda, MD'
}

\begin{abstract}
1Department of Neurological Surgery, University of Pittsburgh Medical Center, Pittsburgh, Pennsylvania; ${ }^{2}$ Department of Otolaryngology, University of Pittsburgh, Pennsylvania; and ${ }^{3}$ Department of Neurosurgery, Taipei Veterans General Hospital, School of Medicine, National Yang-Ming University, Taipei, Taiwan
\end{abstract}

OBJECTIVE The foramen lacerum is a relevant skull base structure that has been neglected for many years. From the endoscopic endonasal perspective, the foramen lacerum is a key structure due to its location at the crossroad between the sagittal and coronal planes. The objective of this study was to provide a detailed investigation of the surgical anatomy of the foramen lacerum and its adjacent structures based on anatomical dissections and imaging studies, propose several relevant key surgical landmarks, and demonstrate the surgical technique for its full exposure with several illustrative cases.

METHODS Ten colored silicone-injected anatomical specimens were dissected using a transpterygoid approach to the foramen lacerum region in a stepwise manner. Five similar specimens were used for a comparative transcranial approach. The osseous anatomy was examined in 32 high-resolution multislice CT studies and 1 disarticulated skull. Representative cases were selected to illustrate the application of the findings.

RESULTS The pterygosphenoidal fissure is the synchondrosis between the lacerum process of the pterygoid bone and the floor of the sphenoid bone. It constantly converges with the posterior end of the vidian canal at a $45^{\circ}$ angle, and its posterolateral end points directly to the lacerum foramen. The pterygoid tubercle separates the vidian canal from the pterygosphenoidal fissure, and forms the anterior wall of the lower part of the foramen lacerum. The lingual process, which forms the lateral wall of the foramen lacerum, was identified in 53 of 64 sides and featured an average height of 5 $\mathrm{mm}$. The mandibular strut separates the foramen lacerum from the foramen ovale and had an average width of $5 \mathrm{~mm}$.

CONCLUSIONS This study provides relevant surgical landmarks and a systematic approach to the foramen lacerum by defining anterior, medial, lateral, and inferior walls that may facilitate its safe exposure for effective removal of lesions while minimizing the risk of injury to the internal carotid artery.

https://thejns.org/doi/abs/10.3171/2018.6.JNS181117

KEYWORDS foramen lacerum; pterygosphenoidal fissure; mandibular strut; Vesalius vein; vidian nerve; translacerum; lacerum ICA; petrous apex; pterygoid tubercle; anatomy

$\mathrm{T}$ HE foramen lacerum represents a key landmark for the endonasal approach due to its location at the crossroad of most surgical routes into the coronal plane.

The foramen lacerum does not represent a true foramen in the sense of an osseous channel containing neurovascular structures, but rather a gap formed by the incomplete confluence of three essential osseous structures composing the central skull base: the sphenoid bone (body and greater wing), temporal bone (petrous apex), and occipital bone (clival part). ${ }^{16}$ Its lower part is filled up with fibrocartilaginous tissue whereas its upper part contains the internal carotid artery (ICA). ${ }^{3}$

In transcranial middle fossa approaches, the foramen lacerum is hidden underneath the Gasserian ganglion and full mobilization and/or transection of V3 and the ganglion is required for its exposure..$^{19}$ In endonasal endoscopic approaches, the vidian canal and nerve have been previ-

ABBREVIATIONS ICA = internal carotid artery; PLL = petrolingual ligament.

SUBMITTED April 24, 2018. ACCEPTED June 28, 2018.

INCLUDE WHEN CITING Published online November 30, 2018; DOI: 10.3171/2018.6.JNS181117. 


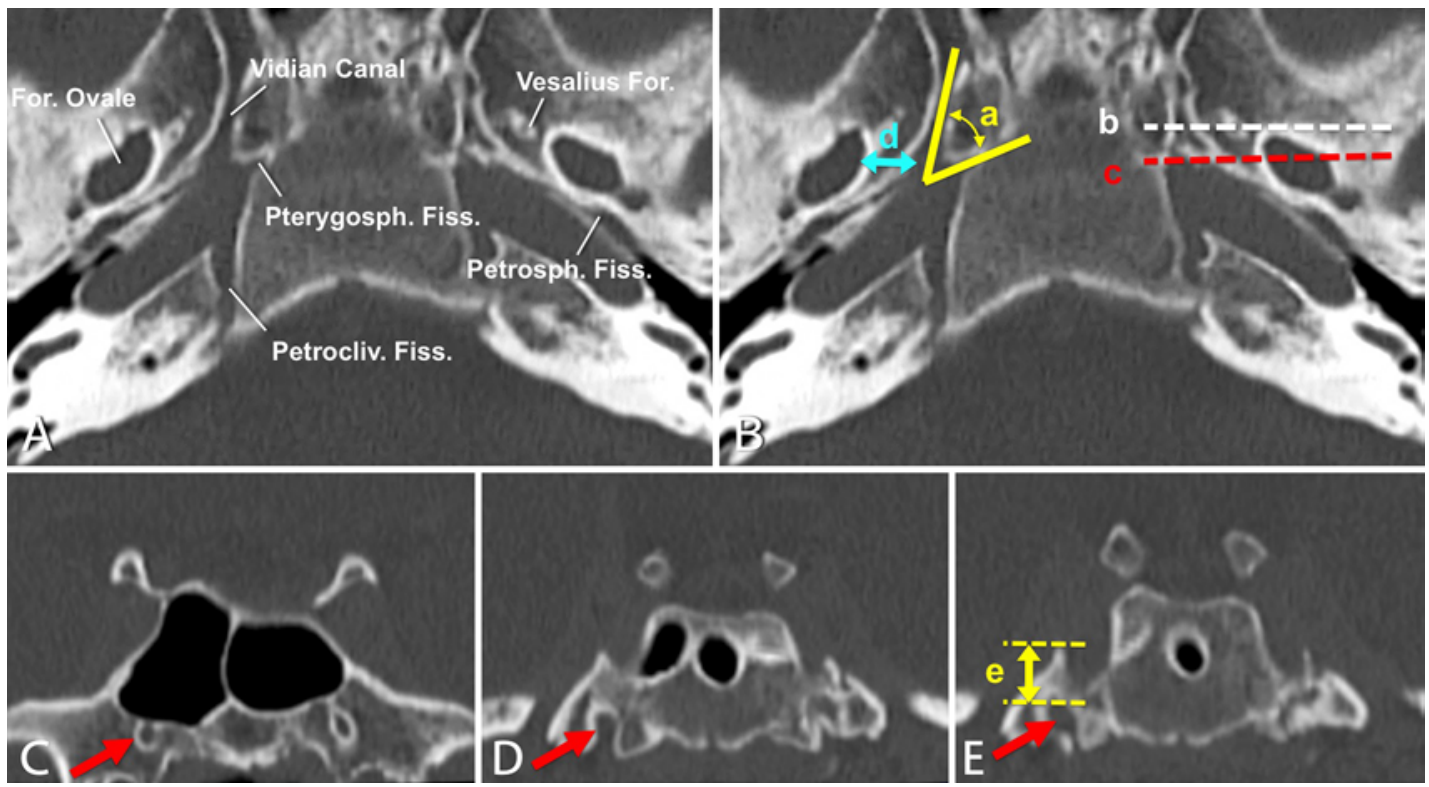

FIG. 1. A and B: Fine-slice CT scans. "a" = the angle between the pterygoid canal and the pterygosphenoidal fissure; "b" (white dashed line) and "c" (red dashed line) = the anterior-most border of the foramen ovale and the foramen lacerum in the axial plane, respectively; "d" = the width of the mandibular strut. C-E: Fine-slice CT scans. The vidian canal (red arrow) is traced from anterior (emergence from the pterygopalatine fossa) to posterior, and the height of the lingual process ("e") is measured at the distal-most point, where the vidian canal enters the foramen lacerum. For. $=$ foramen; Fiss. $=$ fissure; Petrocliv. $=$ petroclival; Petrosph. $=$ petrosphenoidal; Pterygosph. = pterygosphenoidal. Figure is available in color online only.

ously described as reliable landmarks to localize the foramen lacerum. ${ }^{7}$ Following the posterior course of the vidian nerve as it emerges from the pterygopalatine fossa is a useful technique, but this only represents the first and most basic step required for the exposure of the foramen lacerum.

In this study we investigated the surgical anatomy of the foramen lacerum and its adjacent structures using cadaveric dissections and imaging studies, propose several key surgical landmarks, and demonstrate the surgical technique for its full exposure with illustrative cases.

\section{Methods}

\section{Anatomical Dissection}

Ten lightly embalmed human head specimens were prepared for dissection by cannulation of the carotid arteries, vertebral arteries, and internal jugular veins and injected with red and blue silicone. Endoscopic endonasal anatomical dissections were performed using rod lens endoscopes ( $4 \mathrm{~mm}, 18 \mathrm{~cm}$, Hopkins II; $0^{\circ}, 30^{\circ}$, and $45^{\circ}$ ) attached to a high-definition camera and digital video recorder system (Karl Storz). Heads were positioned supine and fixed in a Mayfield head holder to maintain a stable position for navigation and drilling (Stryker).

Four similar specimens were used for comparative transcranial subtemporal approaches and a microscopic dissection of the middle cranial fossa. Another specimen was bisected midsagittally using a high-speed electric saw for a stepwise medial to lateral dissection of the ventral skull base anatomy (magnification $\times 4-40$, surgical microscopes by Zeiss and Mitaka). The osseous anatomy was studied in a disarticulated skull.

\section{Radiological Study}

Thirty-two complete high-resolution multislice CT studies of adult patients $(n=16)$ and anatomical specimens $(n=16)$ without skull base pathology were imported into the OsiriX software (Pixmeo). A thorough review of the osseous anatomy, variations, and spatial relationships between the structures around the foramen lacerum was performed. To recreate the angulation of the anteroposterior axis as encountered in the endoscopic endonasal approach, the axial plane was arranged in a corresponding axis using multiplanar reconstruction. Measurements were obtained for the angle between the pterygoid canal and pterygosphenoidal fissure as well as for the width and height of the mandibular strut and the lingual process, respectively. The spatial relationship between the foramen ovale and foramen lacerum, and the morphology of the Vesalius foramen, were examined (Fig. 1).

\section{Results}

\section{Sphenopetroclival Junction}

The foramen lacerum is bounded anteriorly by the posterior aspect of the pterygoid process (sphenoid bone), posterolaterally by the petrous apex (temporal bone), and posteromedially by the occipital bone (clival part). The respective synchondroses between these osseous structures are the petrosphenoidal fissure (between the petrous bone and sphenoid bone), the petroclival fissure (between the petrous bone and clival part of the occipital bone), and the pterygosphenoidal fissure (between the pterygoid process and the floor of the sphenoid sinus). Specifically, the confluence of these three fissures constitutes the foramen 


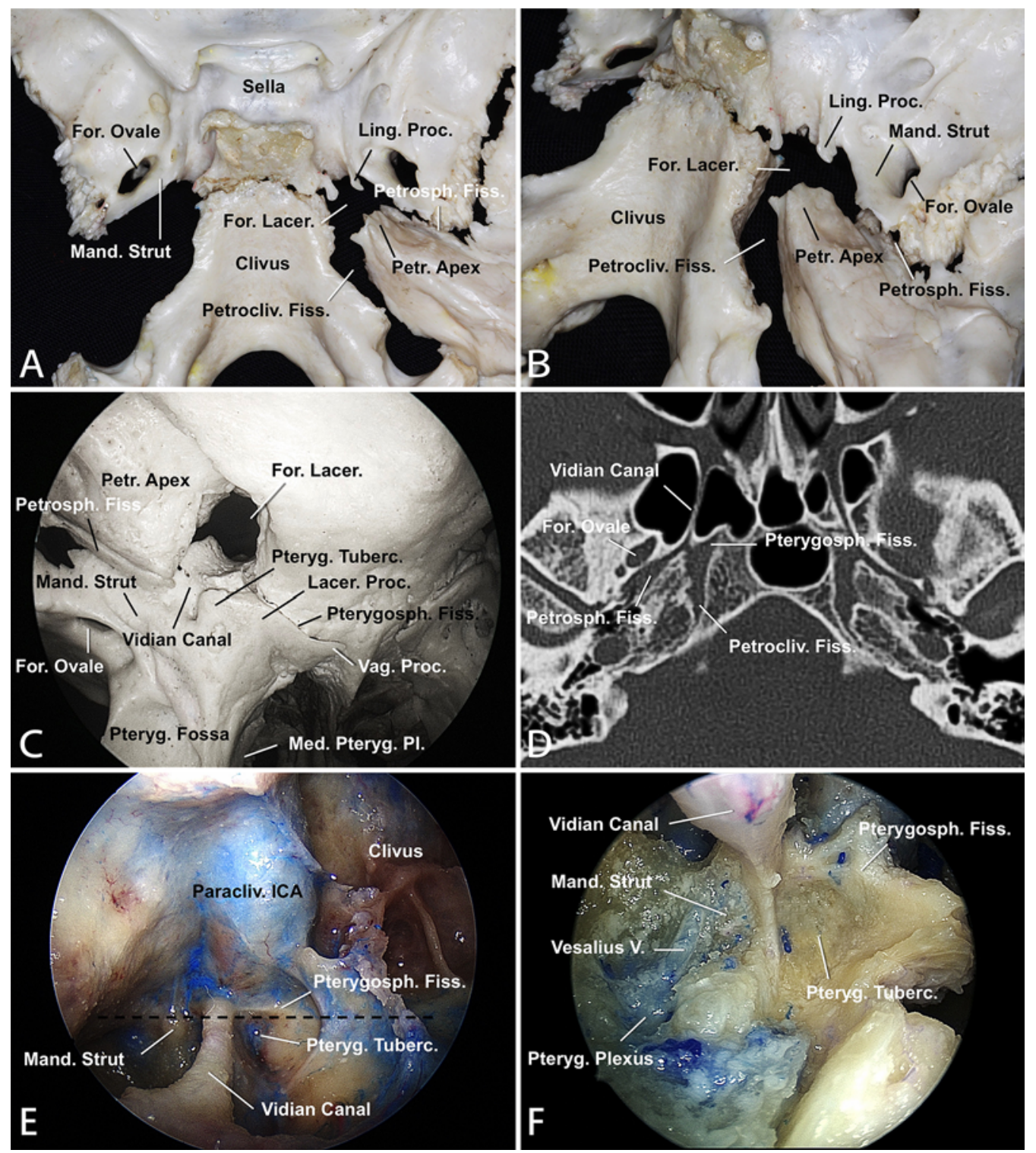

FIG. 2. A and B: Posterior (A) and posterolateral (B) views of the osseous landmarks around the foramen lacerum. The foramen lacerum is formed by the pterygoid process of the sphenoid bone anteriorly, petrous apex of the temporal bone posterolaterally, and the clival part of the occipital bone inferomedially. The articulations between these osseous structures are the petrosphenoidal fissure, petroclival fissure, and pterygosphenoidal fissure. C: Osseous anatomy of the foramen lacerum as seen from a posteroinferior view. On the posterior surface of the pterygoid process, the pterygoid tubercle is a triangular-shaped area located between the vidian canal and the pterygosphenoidal fissure that forms the anterior wall of the lower part of the foramen lacerum. D: Axial bone-window CT scan, corresponding with the same level as the dashed line in panel E. E: Endonasal view into the right foramen lacerum and adjacent structures. The vidian canal, pterygosphenoidal fissure, and pterygoid tubercle can be clearly identified when the lateral recess is well-pneumatized. F: Further drilling exposes the vidian nerve and pterygosphenoidal fissure as they converge at the foramen lacerum, with the pterygoid tubercle between them. For. Lacer. = foramen lacerum; Lacer. Pro. = lacerum process; Ling. Proc. = lingual process; Mand. Strut = mandibular strut; Med. Pteryg. PI. = medial pterygoid plate; Paracliv. ICA = paraclival ICA; Petr. Apex = petrous apex; Pteryg. Tuberc. = pterygoid tubercle; Vag. Proc. $=$ vaginal process; Vesalius $V .=$ Vesalius vein. Copyright Juan C. Fernandez-Miranda (panels A-C, E, and F). Published with permission. Figure is available in color online only.

lacerum, with the pterygosphenoidal fissure running anteromedially, the petroclival fissure running posteromedially, and the petrosphenoidal fissure posterolaterally to the foramen lacerum (Fig. 2).

\section{Pterygoid Process and Vidian Canal}

When inspecting the posterior surface of the pterygoid process, the posterior aspect of the vidian canal appears at the inferolateral aspect of the foramen lacerum. Therefore, the vidian nerve is located slightly inferior and lateral to the lacerum ICA. The pterygoid tubercle is a triangularshaped bone located just inferior and medial to the vidian canal, that separates the vidian canal from the pterygosphenoidal fissure and forms the anterior wall of the lower part of the foramen lacerum (Fig. 2C, E, and F). The medial pterygoid plate continues superiorly to border the vid- 
TABLE 1. Analysis of relevant bone landmarks: measurements in 32 high-resolution CT studies (64 sides) after multiplanar reconstruction in OsiriX

\begin{tabular}{|c|c|c|c|c|}
\hline \multirow[b]{2}{*}{ Landmarks } & \multicolumn{2}{|c|}{ Side $(n=32$ each $)$} & \multicolumn{2}{|c|}{ Percentage or Range } \\
\hline & $\mathrm{Lt}$ & Rt & Lt & Rt \\
\hline Average angle btwn pterygoid canal \& pterygosphenoidal fissure $\left(^{\circ}\right)$ & 45.39 & 45.46 & $36.38-51.41$ & $38.52-51.47$ \\
\hline \multicolumn{5}{|l|}{ Spatial relationship btwn foramen ovale \& foramen lacerum } \\
\hline Anterior to foramen lacerum & 29 & 26 & $90.6 \%$ & $81.25 \%$ \\
\hline Same level as foramen lacerum & 3 & 5 & $9.38 \%$ & $15.63 \%$ \\
\hline Posterior to foramen lacerum & 0 & 1 & $0 \%$ & $3.13 \%$ \\
\hline Presence of Vesalius foramen & 28 & 27 & $87.5 \%$ & $84.36 \%$ \\
\hline \multicolumn{5}{|l|}{ Morphology of Vesalius foramen } \\
\hline Separated & 21 & 22 & $65.63 \%$ & $68.75 \%$ \\
\hline Merged & 7 & 5 & $21.88 \%$ & $15.63 \%$ \\
\hline Average width of mandibular strut (mm) & 5.04 & 5.16 & $3.41-7.03$ & $3.47-7.12$ \\
\hline Presence of lingual process & 27 & 26 & $84.36 \%$ & $81.25 \%$ \\
\hline Average height of lingual process (mm) & 5.40 & 5.54 & $2.41-7.67$ & $3.31-7.34$ \\
\hline
\end{tabular}

ian canal medially and to form the inferomedial aspect of the foramen lacerum; this upper projection of the medial plate can be named the lacerum process of the pterygoid bone, and its synchondrosis with the floor of the sphenoid sinus forms the pterygosphenoidal fissure, a key anatomical landmark (Fig. 2C).

The pterygosphenoidal fissure was consistently observed in all anatomical dissections and CT studies. The vidian canal and the pterygosphenoidal fissure intersect at the anterolateral aspect of the foramen lacerum; in our radiological material, the resulting mean angle was found to be $45^{\circ}$ (Table 1). The medial pterygoid continues anteriorly and medially as the vaginal process of the pterygoid bone and eventually articulates with the sphenoid rostrum and the vomer (Fig. 2C).

\section{Lingual Process and Petrolingual Ligament}

The lingula, or the lingual process of the sphenoid, is a ridge of bone located in the angle between the sphenoid body and its greater wing, along the lateral margin of the carotid groove. In our material, the lingual process was identified in 53 of 64 sides and featured an average height of $5 \mathrm{~mm}$ (Table 1). The petrolingual ligament (PLL) is formed by the periosteal dural layers on either side of the lingual process that fuse at its posterior aspect and continue posteriorly to join the lateral aspect of the petrous apex. The ICA is located medial to the PLL and enters the cavernous sinus at its superior aspect. ${ }^{20}$ The Gasserian ganglion, in turn, lies lateral to the PLL and rests in the trigeminal impression of the petrous apex in the middle cranial fossa floor. In the endonasal middle fossa approach, the posterior aspect of the vidian nerve is located just inferior to both the lingual process and petrolingual ligament. The maxillary branch of the trigeminal nerve (V2) courses lateral to the lingual process within the interdural space of the middle cranial fossa floor as it emerges from the ganglion. ${ }^{1}$ The periosteal dural layer that covers the outer aspect of the lingual process will continue inferiorly with the petrosphenoidal fissure, which runs just lateral and inferior to the lingual process and the PLL (Fig. 3).

\section{Foramen Ovale, Mandibular Strut, and Vesalius Foramen}

The foramen ovale lies lateral to the foramen lacerum; in the anteroposterior axis it was most frequently located just anterior to the foramen lacerum $(n=55)$, rarely found to be at the same level $(n=8)$ and almost never posterior $(n=1)$. Between the foramen lacerum and the foramen ovale, we constantly identified a bony strut that projects in a posterolateral direction to eventually form the medial wall of the foramen ovale. Consistent with the established terminology (e.g., optic or maxillary strut) we introduce the term "mandibular strut" for this bony structure; it serves well as an identifiable surgical landmark (Fig. 4). In our material, the mandibular strut had an average width of $5 \mathrm{~mm}$. The lingual process is located just superior and medial to it and usually projects in a more posterior direction (Fig. 4A). In some cases, an inconsistent sphenoidal emissary foramen (alternatively termed "foramen of Vesalius" or "canaliculus sphenoidalis") can be identified crossing the anterior aspect of the mandibular strut, anterior and medial to the foramen ovale and lateral to the vidian canal (Fig. 4D). It was found to be a clearly defined structure in most specimens $(n=55)$, sometimes it had merged with the foramen ovale $(n=12)$, and rarely it was absent $(n=9)$. When present, the foramen transmits a sphenoidal emissary vein, or Vesalius vein, connecting the pterygoid plexus with the cavernous sinus, which has been classically described as a potential route for retrograde infection. ${ }^{5,8,12,13}$ From the endonasal view, the foramen ovale is identified along the posterior margin of the lateral pterygoid plate up to the infratemporal surface. The Vesalius vein is located anterior to the mandibular strut. From medial to lateral, the anatomical landmarks are: posterior aspect of the vidian nerve and foramen lacerum, mandibular strut (possibly with Vesalius foramen and vein), and foramen ovale (Fig. 4).

\section{Eustachian Tube and Inferior Foramen Lacerum}

The inferior foramen lacerum is filled with fibrocartilaginous tissue that features dense connections to the 

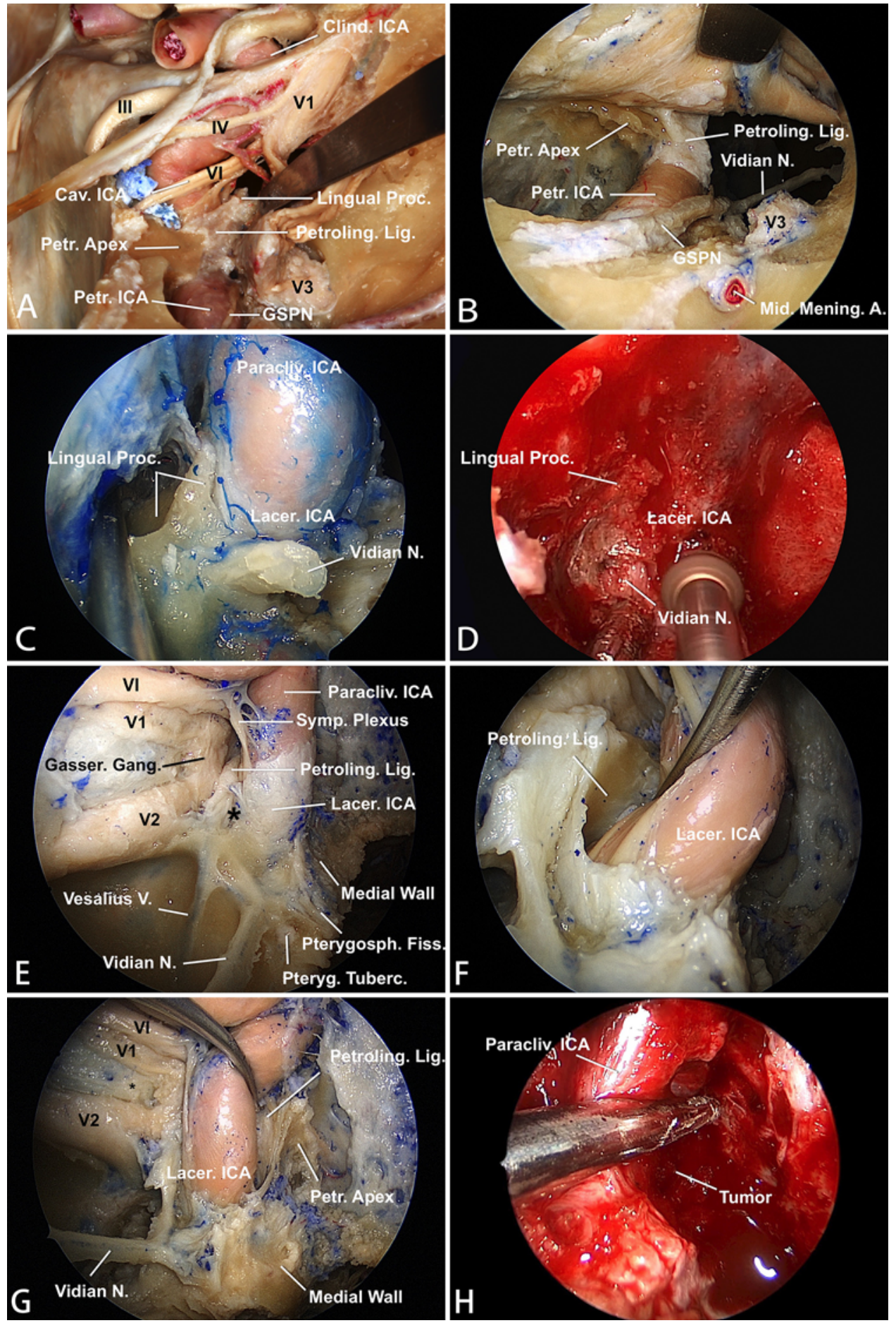

FIG. 3. A: Posterolateral view of the right cavernous sinus and middle fossa. The Gasserian ganglion has been reflected anteriorly to expose the lingual process and the petrolingual ligament. The lacerum ICA is located medial to the petrolingual ligament. B: Posterolateral view in a different specimen; the petrous apex has been partially drilled to expose the petrous ICA segment; V3 has been transected and the Gasserian ganglion reflected upward. The greater superficial petrosal nerve courses anteromedially above the petrous ICA and enters the lower fibrous aspect of the foramen lacerum to continue as the vidian nerve. C: Endonasal close-up view of the right foramen lacerum; the middle fossa dura has been dissected from the lingual process. D: Intraoperative image from illustrative case 2, corresponding to panel C. E: Forty-five degree endoscopic view; the periosteal dura of the carotid canal and middle fossa has been resected. The periosteal dura on both sides of the lingual process merges to form the petrolingual ligament. The asterisk corresponds to the lingual process (removed). The lingual process is located between V2 and the lacerum ICA. F and G: Same specimen as in panel E; the lacerum ICA has been mobilized medially in F and laterally in $\mathrm{G}\left(45^{\circ}\right.$ view) to reveal the attachments of the petrolingual ligament at the lingual process and petrous apex, respectively. FIG. 3. (continued) $\rightarrow$ 
FIG. 3. H: Similar view as panel G from illustrative case 1; the ICA has been mobilized to expose the tumor, the petrous apex was invaded and partially eroded by the tumor. III = oculomotor nerve; IV = trochlear nerve; $\mathrm{VI}$ = abducens nerve; $\mathrm{V} 1$ = ophthalmic branch of trigeminal nerve; $\mathrm{V} 2=$ maxillary branch of trigeminal nerve; $\mathrm{V} 3=$ mandibular branch of trigeminal nerve; Cav. ICA = cavernous ICA; Clind. ICA = clinoid ICA; Gasser. Gang. = Gasserian ganglion; GSPN = greater superficial petrosal nerve; Lacer. ICA $=$ lacerum ICA; Mid. Mening. A. = middle meningeal artery; Petroling. Lig. = petrolingual ligament; Petr. ICA = petrous ICA; Symp. Plexus = sympathetic plexus; Vidian N. = vidian nerve. Copyright Juan C. Fernandez-Miranda (panels A-C, E-G). Published with permission. Figure is available in color online only.

pharyngobasilar fascia inferomedially, the pterygosphenoidal fissure anteriorly, the petroclival fissure inferoposteriorly, and the petrosphenoidal fissure posterolaterally. The eustachian tube has traditionally been divided into a proximal osseous and a distal cartilaginous portion. We previously proposed a surgically oriented classification of the eustachian tube in five segments, from anterior to posterior: nasopharyngeal, pterygoid, lacerum, petrous, and osseous segments. The lacerum segment of the eustachian tube runs just inferior and attaches to the fibrocartilagi-
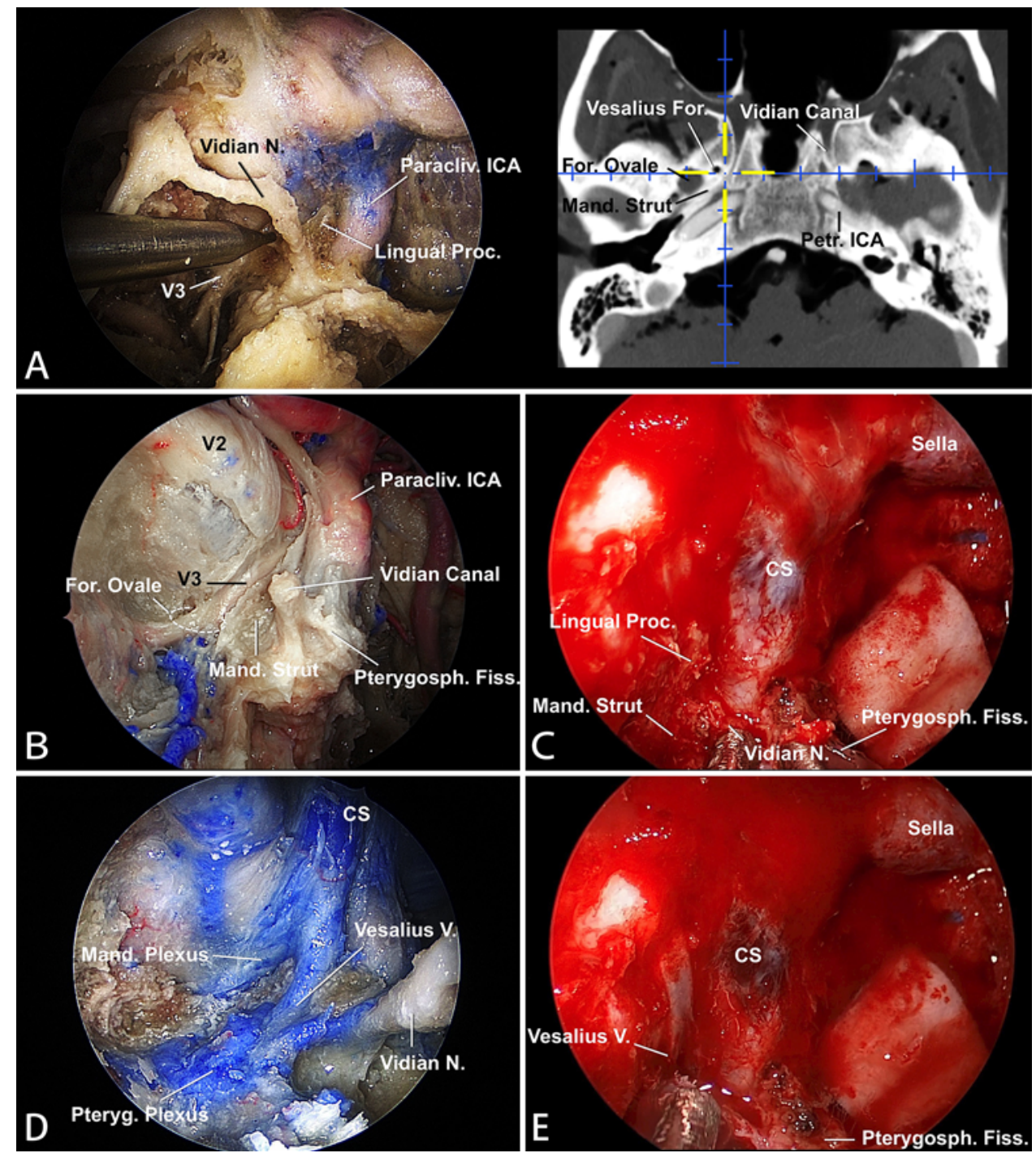

FIG. 4. A: The mandibular strut forms the medial wall of the foramen ovale and is located between the vidian canal and the foramen ovale. CT-guided navigation demonstrates the location of the anterior surface of the mandibular strut. B: The vidian nerve, mandibular strut, and foramen ovale are seen from medial to lateral, respectively. C: Intraoperative view from illustrative case 2 , corresponding to panel B. D: Venous plexuses lateral to the foramen lacerum; the Vesalius vein is one of the sphenoidal emissary veins connecting the cavernous sinus with the pterygoid venous plexus. The Vesalius vein is usually located anteromedially to the foramen ovale; this location corresponds to the anterior aspect of the mandibular strut. E: The Vesalius vein was identified and coagulated in illustrative case 2 . CS = cavernous sinus; Mand. Plexus = mandibular venous plexus; Pteryg. Plexus = pterygoid venous plexus; Vesalius For. = Vesalius foramen. Copyright Juan C. Fernandez-Miranda (panels A, B, and D). Published with permission. Figure is available in color online only. 

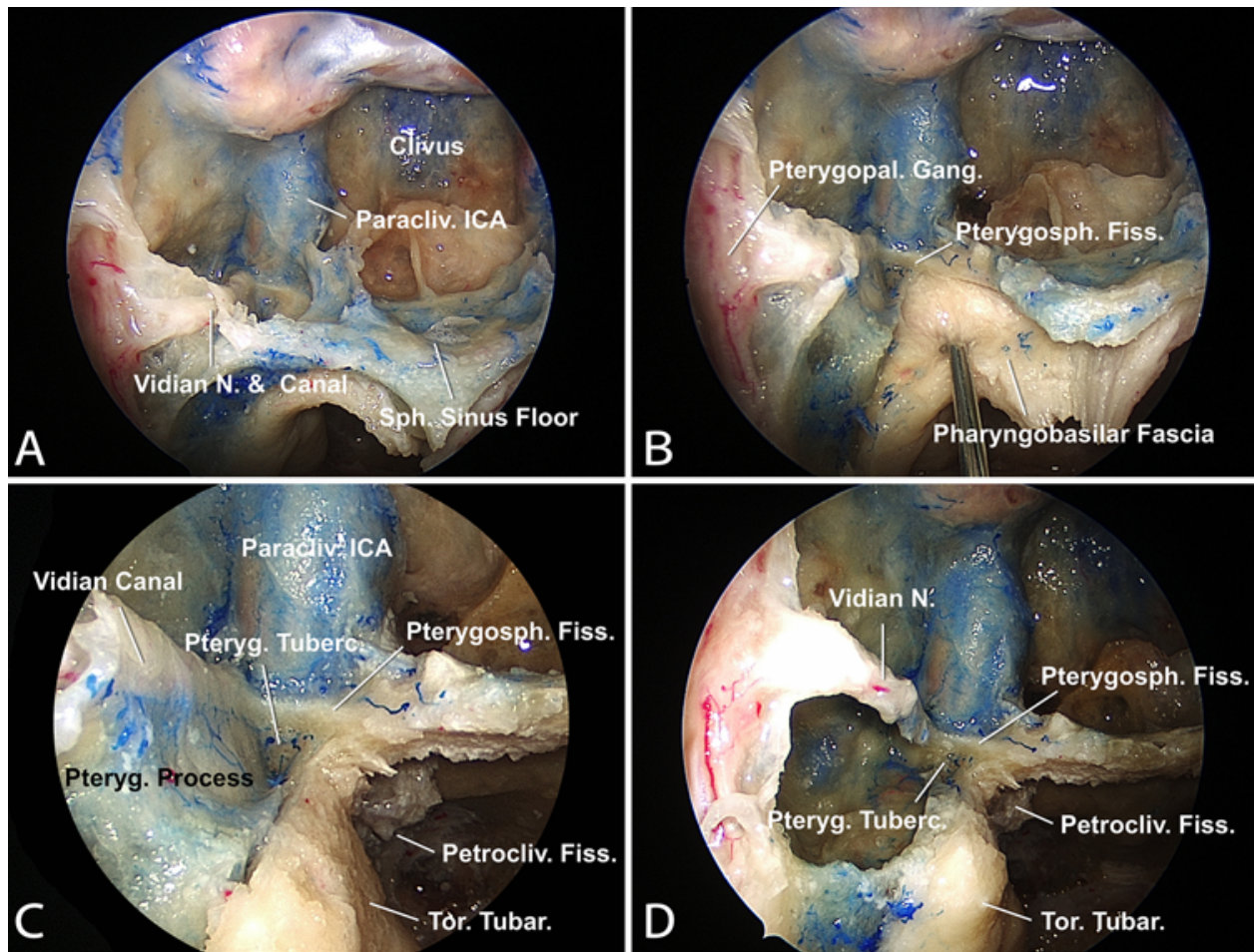

FIG. 5. Stepwise endonasal dissection showing the fibrocartilaginous tissues at the inferior aspect of the foramen lacerum. A: The lateral recess has been exposed via a transpterygoid approach. B: The floor of the right sphenoid sinus has been partially resected to expose the pharyngobasilar fascia attached to the medial aspect of the pterygosphenoidal fissure. C: The confluence of the petroclival and pterygosphenoidal fissures leads to the inferomedial aspect of the foramen lacerum where the torus tubarius has an attachment site as well. The pterygoid tubercle is situated below the pterygosphenoidal fissure to form the anterior wall of the foramen lacerum. D: The vidian nerve is located inferolaterally of the lacerum ICA; the lateral-most aspect of the pterygosphenoidal fissure also leads to the distal end of the vidian canal. Pterygopal. Gang. = pterygopalatine ganglion; Pteryg. Process = pterygoid process; Sph. Sinus Floor = sphenoid sinus floor; Tor. Tubar. = torus tubarius. Copyright Juan C. Fernandez-Miranda. Published with permission. Figure is available in color online only.

nous portion of the foramen lacerum. ${ }^{9,10}$ Despite the lack of osseous landmarks, these constant soft tissues still provide reliable guidance for exploration of the inferior aspect of the foramen lacerum (Fig. 5).

\section{Endonasal Surgical Approach to the Foramen Lacerum Anterior Wall: Vidian Canal and Pterygosphenoidal Fissure}

To expose the foramen lacerum, a wide maxillary antrostomy and sphenoidotomy are required in early stages. Resection of the thin posterior wall of the maxillary sinus provides access to the pterygopalatine fossa. The sphenoid process of the palatine bone is identified and removed, which exposes the palatosphenoidal artery, a reliable landmark for localization of the vidian nerve."1

Drilling begins at the floor of the sphenoid sinus and the pterygoid process. The removal of the floor of the sphenoid sinus and vaginal process will expose the medial aspect of the pterygosphenoidal fissure, and the drilling of the lacerum process of the medial pterygoid plate (inferomedial to the vidian canal) will expose its lateral aspect. The fibrous tissue of the pterygosphenoidal fissure provides an excellent surgical landmark that can be followed from anterior to posterior, from medial to lateral, and from inferior to superior until the medial and lower aspects of the foramen lacerum are encountered. The vidian canal and the pterygosphenoidal fissure converge posteriorly at a $45^{\circ}$ angle, and as drilling progresses in between them, the triangular-shaped pterygoid tubercle will remain between the pterygosphenoidal fissure and the vidian canal to form the anterior wall of the lower part of the foramen lacerum. Removal of the pterygoid tubercle exposes the fibrocartilaginous tissue of the lower aspect of the foramen lacerum, and the corresponding ICA segment will be just above. The vidian nerve has to be sacrificed and transected if a more extensive lateral exposure is required. The resulting nerve stump can then serve as a landmark, given its constant localization just lateral to the anterior wall of the foramen lacerum, inferolateral to the lacerum ICA, inferior to the lingual process, and inferomedial to V2 (Fig. 6).

\section{Medial Wall: Pterygosphenoidal and Petroclival Fissures}

For most approaches that require exposure of the foramen lacerum, the middle portion of the clivus has to be completely removed by drilling the floor of the sphenoid sinus down to the level of the pharyngobasilar fascia attachment. The bone covering the paraclival ICA is also gently skeletonized to allow for its maximal mobilization and to access the medial petrous apex located just posterior. In general, it is safer to start the skeletonization of the paraclival ICA by exposing the dura of the anterior wall of the cavernous sinus superiorly. This provides a reli- 

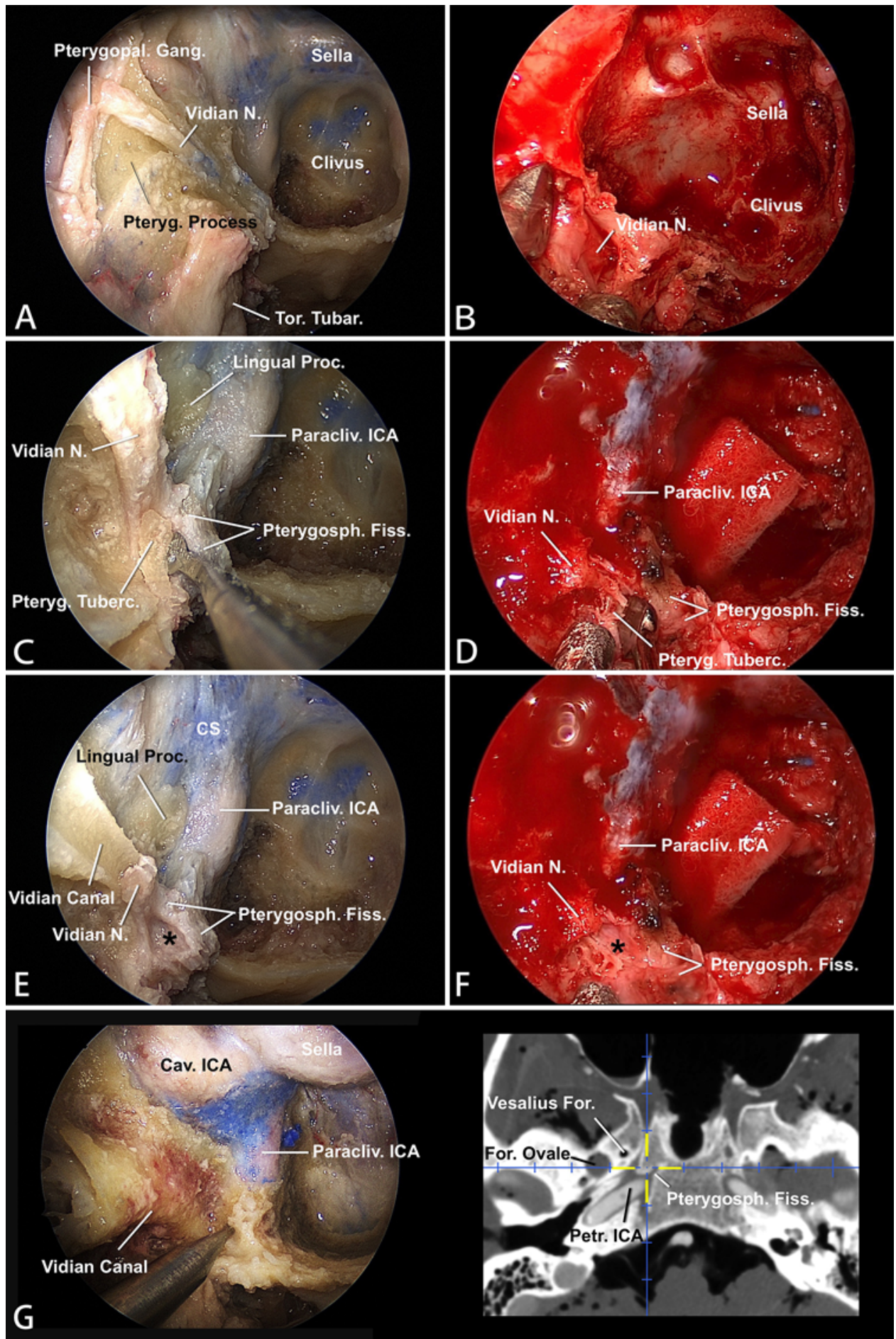

FIG. 6. Surgical approach to the anterior wall of the foramen lacerum. A: The pterygopalatine fossa has been opened to expose the pterygopalatine ganglion. The vidian nerve is localized as it emerges from the ganglion and enters the vidian canal. B: Similar view as panel A; the vidian nerve is identified in illustrative case 1. C: The pterygosphenoidal fissure has been exposed, and the pterygoid tubercle is identified as it forms the anterior wall of the foramen lacerum. D: The pterygoid tubercle in illustrative case 2 is identified. E: The pterygoid tubercle has been removed. The asterisk represents the safe entry zone into the inferior foramen lacerum region. F: Similar view as panel E from illustrative case 2. G: CT-guided navigation demonstrates the location of the anterior wall of foramen lacerum. Copyright Juan C. Fernandez-Miranda (panels A, C, E, and G). Published with permission. Figure is available in color online only. 

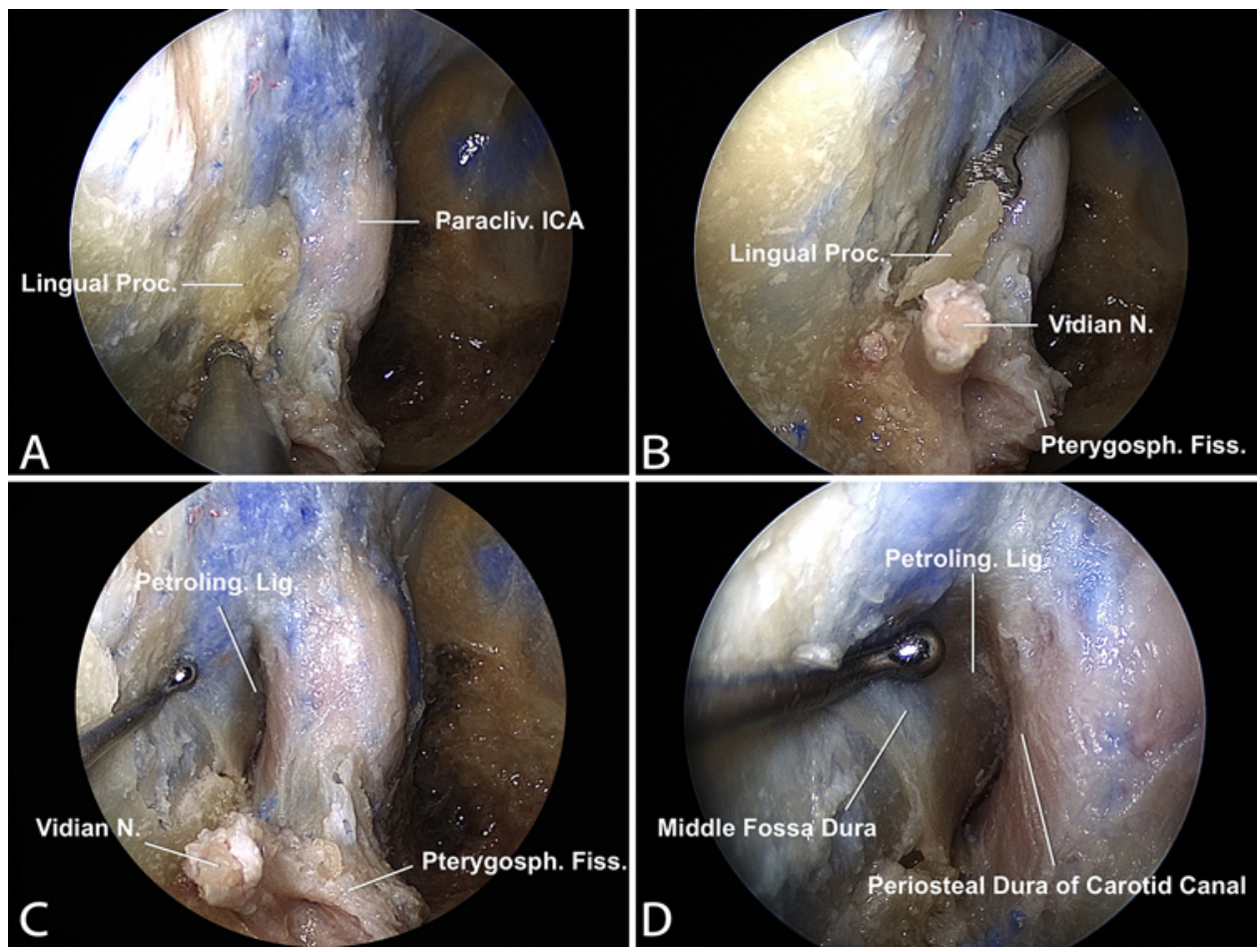

FIG. 7. Surgical approach to the lateral wall of the foramen lacerum. A: The lingual process forms the lateral wall of the foramen lacerum. To facilitate the detachment of the lingual process, drilling begins at its base. B: The lingual process can be fractured following careful dissection of the dural attachments on both sides. C: The lingual process has been completely removed to expose the petrolingual ligament behind. D: Close-up view of panel C; the dura attached on both sides of the lingual process merges posteriorly to form the petrolingual ligament. Copyright Juan C. Fernandez-Miranda. Published with permission. Figure is available in color online only.

able plane for continuing the uncovering of the paraclival ICA in a caudal direction toward the foramen lacerum. The clival bone immediately posterior and medial to the pterygosphenoidal fissure forms the medial wall of the foramen lacerum and can be removed posteriorly up to the level of the petroclival fissure. Complete removal of this bone exposes the medial aspect of the lacerum ICA as it emerges from the petrous carotid canal (Figs. 3E and G, and $6 \mathrm{E})$.

\section{Lateral Wall: Lingual Process and Petrolingual Ligament}

Maximal mobilization of the paraclival ICA requires further removal of bone lateral to the ICA. The lingual process of the sphenoid bone is located between V2 and the lacerum ICA, and forms the lateral wall of the foramen lacerum (Fig. 3E). In a first step, the base of the lingual process, located superior and slightly lateral to the posterior end of the vidian canal, is drilled to facilitate its detachment. After carefully dissecting free the dural attachments on both sides (corresponding to the petrolingual ligament and middle fossa dura), the lingual process can be fractured and removed in a superior to inferior trajectory (Fig. 7). It is of paramount importance to carefully mobilize the lingual process prior to its final removal to minimize the risk of ICA injury. If further lateral exposure is required, drilling will progress from the lateral aspect of the vidian canal into the mandibular strut. Occasional venous bleeding can be encountered from an emissary vein connecting the cavernous sinus with the pterygoid plexus (Vesalius vein). The foramen ovale will eventually be exposed by drilling lateral to the mandibular strut, which serves as a constant surgical landmark (Fig. 4B).

\section{Inferior Wall: Eustachian Tube}

For exposure of the inferior wall of the foramen lacerum, safe mobilization of the eustachian tube is required. The pterygosphenoidal fissure is first identified as a key landmark. The pharyngobasilar fascia along with the mucosa above the torus tubarius is detached and removed from the inferior surface of the sphenoid sinus floor and medial pterygoid plate until the tight insertion of the pharyngobasilar fascia into the clival bone is reached. Further dissection detaches the nasopharyngeal and pterygoid segments of the eustachian tube. The pterygosphenoidal fissure serves as the superior border of a safe entry zone to the inferior foramen lacerum region. Transecting the pterygosphenoidal fissure tissues below this border detaches the lacerum segment of the eustachian tube from the inferior foramen lacerum wall without ICA injury (Fig. 7D). This provides a sublacerum corridor into the inferior aspect of the petrous apex, which forms the floor of the foramen lacerum, and toward the ventral aspect of the petroclival fissure and jugular foramen.

\section{Case Illustration 1: Chondrosarcoma}

A 74-year-old woman presented with mild diplopia secondary to partial right abducens nerve palsy. Imaging findings were compatible with chondrosarcoma centered 
on the right petroclival fissure and foramen lacerum, with inferior extension into the jugular foramen and superior extension into the petrous apex. The petrous and lacerum segments of the ICA were displaced laterally by the tumor mass. An endoscopic endonasal approach was performed (Video 1), applying the anatomical landmarks and surgical nuances described above, and achieving a near-total (> 95\%) resection with no complications and recovery from abducens nerve palsy at 3 months follow-up.

VIDEO 1. Clip showing an endoscopic endonasal approach performed to remove a chondrosarcoma centered on the right petroclival fissure and foramen lacerum, with inferior extension into the jugular foramen and superior extension into the petrous apex. The anatomical landmarks and surgical nuances to safely expose the foramen lacerum are demonstrated. GKS = Gamma Knife surgery. Copyright Juan C. Fernandez-Miranda. Published with permission. Click here to view.

Pathology confirmed chondrosarcoma grade II with a Ki67 index of 1\%. Gamma Knife radiosurgery has been recommended for treatment of residual tumor at the jugular foramen.

\section{Case Illustration 2: Chordoma}

A 37-year-old man with new onset of progressive palsy of the sixth cranial nerve and a skull base lesion compatible with clival chordoma underwent partial resection at an outside institution via a transcranial approach, with significant residual tumor at the clivus, dorsum sella, posterior clinoids, and petrous apex; extensive dural invasion; and intradural extension with attachment to the basilar artery and its long perforating branches. Gross-total resection was achieved using an endoscopic endonasal transclival approach, ipsilateral transpterygoid approach to the foramen lacerum for carotid artery mobilization, bilateral interdural transcavernous approach with posterior clinoidectomies, and contralateral transmaxillary approach to the petrous apex (Video 2).

\section{VIDEO 2. Clip showing an endoscopic endonasal transclival}

approach used to remove a residual chordoma at the clivus, dorsum sella, posterior clinoids, and petrous apex. The surgical techniques include an ipsilateral transpteryoid approach to the foramen lacerum for carotid artery mobilization, bilateral interdural transcavernous approach with posterior clinoidectomies, and contralateral transmaxillary approach to the petrous apex. $\mathrm{CN}=$ cranial nerve. Copyright Juan C. Fernandez-Miranda. Published with permission. Click here to view.

Reconstruction was performed in a multilayer fashion with fascia lata and fat grafts, and an extended nasoseptal flap; a lumbar drain was placed for 3 days. No CSF leak occurred, and the abducens nerve palsy significantly improved at 3 months follow-up. The patient underwent adjuvant proton beam radiation therapy.

\section{Discussion}

The foramen lacerum was introduced to the anatomical literature in the 19th century by Gruber. ${ }^{4}$ Yet, lacking a detailed description of its surgical anatomy and clinical relevance, the foramen lacerum remained a relevant skull base structure and was neglected for many years. ${ }^{1}$ Several skull base lesions centered in the foramen lacerum region, most typically chondrosarcomas, but essentially all other pathologies affecting the cavernous sinus, Meckel's cave, petrous apex, clival and petroclival region, nasopharyngeal region and eustachian tube, pterygopalatine fossa, infratemporal fossa, and jugular foramen may necessitate exposure of the foramen lacerum. In the traditional microsurgical approach, the foramen lacerum is hidden medial to the trigeminal ganglion and remains difficult to access. The medial-to-lateral trajectory of the endonasal approach, however, provides direct access to the foramen lacerum, but its safe and effective exposure requires intimate knowledge of its surgical anatomy. In this paper we describe key anatomical landmarks that have proven useful in our surgical experience, and we provide a rational approach to understanding the complex anatomy of the foramen lacerum by defining anterior, medial, lateral, and inferior walls, and how to remove them.

Drilling the inferior $180^{\circ}$ of the vidian canal has become a well-established technique to help identify the lacerum segment of the ICA. ${ }^{718}$ In this paper, we propose the pterygosphenoidal fissure as an additional key landmark for safe and effective exposure of the foramen lacerum. When performing surgery, this fissure has usually been ignored or considered part of the connective tissues around the inferior foramen lacerum. From the results of our anatomical study, the pterygosphenoidal fissure is one of the three fissures that constitute the foramen lacerum; because it is the one located most anteriorly, it is ideally suited as a surgical landmark when performing anterior approaches, such as the endoscopic endonasal approach. In our observations, this fissure constantly converges with the posterior end of the vidian canal at a $45^{\circ}$ angle, and its posterolateral end points directly to the lacerum foramen. As a result, early identification of this landmark and its exposure by drilling the sphenoid sinus floor medially and the pterygoid process laterally has become routine in our surgical practice for surgical exposure of the foramen lacerum, minimizing the risk of accidental injury to the lacerum ICA. Here we introduce the pterygoid tubercle and the lacerum process of the medial pterygoid as key landmarks when exposing the anterior and medial walls of the lower foramen lacerum, respectively.

From the endonasal endoscopic perspective, the lateral aspect of the foramen lacerum is narrow and difficult to access, particularly when the pterygoid or lateral recess is poorly pneumatized. The surgical landmarks in this region are the lingual process and the mandibular strut. Different morphologies of the lingual process have been described previously. ${ }^{16}$ In our investigation, we outline the surgical technique for safe removal (or reduction) of the lingual process. This maneuver facilitates lateral mobilization of the ICA to access to the medial petrous apex, and medial mobilization of the ICA when access to the middle cranial fossa is needed. The "quadrangular space" has been described as the "front door" to Meckel's cave. ${ }^{6}$ In a more recent study, the authors defined the "strut of the quadrangular space," which actually corresponds to and is more accurately defined as the lingual process of the sphenoid bone. ${ }^{2}$ Removal of the lingual process is key to open this space, which provides access to the middle cranial fossa, specifically to the posterior aspect of the anteromedial and anterolateral triangles of the middle fossa as they converge 
into Meckel's cave, the accurate anatomical correlate of the "quadrangular space."

The designation of "strut" should be reserved for bony structures between foramina. Consequently, in this paper we introduce the term "mandibular strut." Similar to the maxillary strut separating the superior orbital fissure from the foramen rotundum, the mandibular strut separates the foramen ovale from the vidian canal and foramen lacerum. From the endoscopic perspective, the mandibular strut is lateral to the posterior end of the vidian canal and a reliable landmark to localize the mandibular nerve. In our experience, occasional venous bleeding is encountered during drilling around the mandibular strut due to the presence of the emissary vein of Vesalius that (when present) passes through the Vesalius foramen in the anterior aspect of the mandibular strut. The vein of Vesalius, which connects the cavernous sinus with the pterygoid plexus, should be coagulated when the approach is extended along the floor of the middle fossa from the foramen lacerum to the foramen ovale. Both foramina are typically located at almost the same anteroposterior level but the foramen ovale is slightly more anterior than the foramen lacerum in most cases.

The lacerum segment of cartilaginous eustachian tube attaches superiorly to the inferior portion of the foramen lacerum. In order to expose the inferior petrous apex and ventral jugular foramen, disconnection of the cartilaginous eustachian tube from the foramen lacerum fibrocartilage is required to develop a translacerum or sublacerum route. ${ }^{14,15,17}$ There are two previous case reports on this technique, and we have recently reported the clinical application and outcome of this technique for lesions involving the ventral jugular foramen in a series of 14 patients. However, a description of constant landmarks and safe boundaries to disconnect the eustachian tube from the foramen lacerum was still lacking. In this study, we report the fibrous tissue of the pterygosphenoidal fissure to be a constant and reliable landmark: its inferior aspect will continue with the confluence of the petroclival synchondrosis and its superior aspect will lead directly to the foramen lacerum. Therefore, the pterygosphenoidal fissure defines the safe entry zone to disconnect the cartilaginous eustachian tube and expose the inferior aspect of the petrous apex at the floor of the foramen lacerum.

Partial or full exposure of the foramen lacerum is required for effective resection of different types of skull base lesions. Skull base chondrosarcomas characteristically arise from one of the synchondroses that constitute the foramen lacerum, most commonly the petroclival fissure. They expand the foramen lacerum and may extend superiorly toward the petrous apex, middle fossa, and cavernous sinus, and inferiorly toward the jugular foramen, cerebellopontine and cerebellomedullary cisterns, and parapharyngeal space. Clival chordomas often extend laterally and inferiorly toward the petrous apex and foramen lacerum, requiring a sublacerum approach for complete resection of the petrous apex tumor component. Petroclival meningiomas, while rarely resected via the endonasal approach and not directly involving the foramen lacerum, will benefit from full exposure of the paraclival and lacerum ICA segments to maximize surgical access. Middle fossa lesions, such as trigeminal schwannomas, may require removal of the lateral wall of the foramen lacerum to optimize the endonasal surgical corridor. Pterygopalatine fossa lesions, such as juvenile angiofibromas and adenoid cystic carcinomas, may extend posteriorly toward the foramen lacerum. Nasopharyngeal carcinomas, while rarely requiring resection, will have their superolateral limit of resection defined by the foramen lacerum. All these cases highlight the relevance of the foramen lacerum in skull base surgery and the importance of an intimate knowledge of its surgical anatomy as described here.

\section{Conclusions}

The development and refinement of endoscopic endonasal surgery has brought significant relevance to the foramen lacerum when treating a variety of skull base lesions. In this study, we describe novel and practical anatomical surgical landmarks for safe and effective exposure of the foramen lacerum, such as the pterygosphenoidal fissure, the pterygoid tubercle, the lacerum process of the pterygoid bone, the mandibular strut, and the lingual process of the sphenoid bone. Additionally, we provide a systematic approach to the foramen lacerum by defining anterior, medial, lateral, and inferior walls that may facilitate its safe exposure for effective removal of lesions while minimizing the risk of injury to the ICA.

\section{References}

1. Abhinav K, Panczykowski D, Wang WH, Synderman CH, Gardner PA, Wang EW, et al: Endoscopic endonasal interdural middle fossa approach to the maxillary nerve: anatomic considerations and surgical relevance. Oper Neurosurg (Hagerstown) 13:522-528, 2017

2. Dolci RLL, Ditzel Filho LFS, Goulart CR, Upadhyay S, Buohliqah L, Lazarini PR, et al: Anatomical nuances of the internal carotid artery in relation to the quadrangular space. J Neurosurg 128:174-181, 2018

3. Fernandez-Miranda JC: Intracranial region, in Standring S (ed): Gray's Anatomy, ed 41. Amsterdam: Elsevier, 2016, pp 429-442

4. Gruber W: Beiträge zur Anatomie des Schadelgrundes. Mem Akad Imp Sci St Petersburgh 13 Suppl:S1-S34, 1869

5. Gupta N, Ray B, Ghosh S: Anatomic characteristics of foramen vesalius. Kathmandu Univ Med J (KUMJ) 3:155-158, 2005

6. Kassam AB, Prevedello DM, Carrau RL, Snyderman CH, Gardner P, Osawa S, et al: The front door to Meckel's cave: an anteromedial corridor via expanded endoscopic endonasal approach- technical considerations and clinical series. Neurosurgery 64 (3 Suppl):ons71-ons83, 2009

7. Kassam AB, Vescan AD, Carrau RL, Prevedello DM, Gardner P, Mintz AH, et al: Expanded endonasal approach: vidian canal as a landmark to the petrous internal carotid artery. $\mathbf{J}$ Neurosurg 108:177-183, 2008

8. Lanzieri CF, Duchesneau PM, Rosenbloom SA, Smith AS, Rosenbaum AE: The significance of asymmetry of the foramen of Vesalius. AJNR Am J Neuroradiol 9:1201-1204, 1988

9. Liu J, Pinheiro-Neto CD, Fernandez-Miranda JC, Snyderman $\mathrm{CH}$, Gardner PA, Hirsch BE, et al: Eustachian tube and internal carotid artery in skull base surgery: an anatomical study. Laryngoscope 124:2655-2664, 2014

10. Ozturk K, Snyderman CH, Gardner PA, Fernandez-Miranda JC: The anatomical relationship between the eustachian 
tube and petrous internal carotid artery. Laryngoscope 122:2658-2662, 2012

11. Pinheiro-Neto CD, Fernandez-Miranda JC, Rivera-Serrano CM, Paluzzi A, Snyderman CH, Gardner PA, et al: Endoscopic anatomy of the palatovaginal canal (palatosphenoidal canal): a landmark for dissection of the vidian nerve during endonasal transpterygoid approaches. Laryngoscope 122:612,2012

12. Raval BB, Singh PR, Rajguru J: A morphologic and morphometric study of foramen Vesalius in dry adult human skulls of Gujarat region. J Clin Diagn Res 9:AC04-AC07, 2015

13. Shinohara AL, de Souza Melo CG, Silveira EM, Lauris JR, Andreo JC, de Castro Rodrigues A: Incidence, morphology and morphometry of the foramen of Vesalius: complementary study for a safer planning and execution of the trigeminal rhizotomy technique. Surg Radiol Anat 32:159-164, 2010

14. Simal-Julián JA, Miranda-Lloret P, Beltrán-Giner A, PlazaRamirez E, Botella-Asunción C: Full endoscopic endonasal extreme far-medial approach: Eustachian tube transposition. J Neurosurg Pediatr 11:584-590, 2013

15. Taniguchi M, Akutsu N, Mizukawa K, Kohta M, Kimura H, Kohmura E: Endoscopic endonasal translacerum approach to the inferior petrous apex. J Neurosurg 124:1032-1038, 2016

16. Tauber M, van Loveren HR, Jallo G, Romano A, Keller JT: The enigmatic foramen lacerum. Neurosurgery 44:386-393, 1999

17. Vaz-Guimaraes F, Nakassa ACI, Gardner PA, Wang EW, Snyderman CH, Fernandez-Miranda JC: Endoscopic endonasal approach to the ventral jugular foramen: anatomical basis, technical considerations, and clinical series. Oper Neurosurg (Hagerstown) 13:482-491, 2017

18. Vescan AD, Snyderman CH, Carrau RL, Mintz A, Gardner P, Branstetter B IV, et al: Vidian canal: analysis and relationship to the internal carotid artery. Laryngoscope 117:13381342,2007

19. Yasuda A, Campero A, Martins C, Rhoton AL Jr, de Oliveira E, Ribas GC: Microsurgical anatomy and approaches to the cavernous sinus. Neurosurgery 62 (6 Suppl 3):1240-1263, 2008

20. Ziyal IM, Salas E, Wright DC, Sekhar LN: The petrolingual ligament: the anatomy and surgical exposure of the posterolateral landmark of the cavernous sinus. Acta Neurochir (Wien) 140:201-205, 1998

\section{Disclosures}

The authors report no conflict of interest concerning the materials or methods used in this study or the findings specified in this paper.

\section{Author Contributions}

Conception and design: Fernandez-Miranda. Acquisition of data: WH Wang, Lieber, Mathias, Sun. Analysis and interpretation of data: Fernandez-Miranda, WH Wang, Lieber, Mathias, Sun. Drafting the article: WH Wang. Critically revising the article: Fernandez-Miranda, WH Wang, Lieber. Reviewed submitted version of manuscript: Fernandez-Miranda, WH Wang, Lieber. Approved the final version of the manuscript on behalf of all authors: Fernandez-Miranda. Administrative/technical/material support: Fernandez-Miranda, WH Wang, Gardner, Snyderman, EW Wang.

\section{Supplemental Information \\ Videos \\ Video 1. https://vimeo.com/285104805. \\ Video 2. https://vimeo.com/285104881.}

\section{Correspondence}

Juan C. Fernandez-Miranda: Stanford University, Palo Alto, CA. drjfm@stanford.edu. 